\title{
Nonlinear Distortion Effects In Generalized Frequency Division Multiplexing
}

\author{
M.Kaviya, S.Sumathi
}

\begin{abstract}
Generalised Frequency Division Multiplexing (GFDM) is utilized in the fifth era cell organize as opposed to the Orthogonal Frequency Division Multiplexing (OFDM) since GFDM has numerous appealing highlights like heartiness towards recurrence particular blurring, simple execution and sensible, and so forth. GFDM has just a single cyclic prefix (CP) for a lot of images in a period OFDM it uses a solitary $C P$ per image. Right now, research the effects of nonlinear mutilation on the different information numerous yield (MIMO)- GFDM framework when the sign is passed the HPA, which is displayed with sufficiency and stage contortion. The execution of the proposed strategy is resolved as far as range investigation, PAPR examination, and bit blunder rate (BER) investigation. The recreation results show that the proposed plan prevails with regards to making up for nonlinear contortions brought about by the HPA for huge contribution back-off(IBO) values.

Keywords: Generalized Frequency Division Multiplexing, Interference, Matched Filter, Zero Forcing, High Power Amplifier.
\end{abstract}

\section{INTRODUCTION}

The wireless communication frameworks center around transmitting voice, video, and information in the neighborhood and wide zones. A piece of remote correspondence progressively needs to interface with systems, perform questions, move messages and records and even take an interest in shared figuring. Wireless communication has been generally utilized for its phenomenal highlights. [1] The growing solicitation of remote correspondence has achieved as far as possible burden altering in which the structure will adequately move customers from the one section to another point reliant on as far as possible. It is basic to improve the incorporation zone without updating or manufacture another framework at some point later. Even though remote correspondence has numerous preferences over wired there are barely any difficulties looked in it. [2]The primary difficulties happen in the physical channel of correspondence frameworks. A portion of the components is a multipath signal reflection, low sign quality, obstruction and so on. The issue is to defeat the twisting of got flags by time fluctuating and recurrence specific spread way.

Revised Manuscript Received on March 27, 2020.

*Correspondence Author Engineering, Hosur,Tamilnadu,India. Email: kaviyam51996@gmail.com

Dr.S.Sumathi, Professor \& Head,Dept of ECE, Adhiyamaan College of Engineering,Hosur,Tamilnadu,India.Email:sumathi_2005@rediffmail.com

(c) The Authors. Published by Blue Eyes Intelligence Engineering and Sciences Publication (BEIESP). This is an open access article under the CC BY-NC-ND license (http://creativecommons.org/licenses/by-nc-nd/4.0/)
M.Kaviya*, Communication Systems, Adhiyamaan College of

[3] Various issues went up against are power use and multifaceted nature of programming. Various strategies are used to vanquish these weights. [4] Orthoganol Frequency Division Multiplexing (OFDM) is a technique that is used in both wired and remote system. This is an electronic change strategy wherein a sign is a piece of a couple narrowband channels. Completely any sort of OFDM is Long Term Evolution (LTE), Voice over Long-Term Evolution strategy (VoLTE, etc. VoLTE is the OFDM strategy that is used in starting late used system, principally as a result of its quality towards repeat decision. [5] The principal points of interest of OFDM is insusceptibility to specific blurring, protection from obstruction, range productivity, opposition towards Inter image impedance (ISI), protection from narrowband impact, less complex channel adjustment, low affectability to time synchronization blunder, etc. OFDM can be effortlessly adjusted to cut off channel conditions without complex time-space evening out. The weaknesses of OFDM are, that they experience the ill effects of out-of-band (OOB) emanation brought about by rectangular heartbeat forming, this expands the impedance in the framework and the potential for misusing non-adjacent range lumps in procedures like bearer collection. This is the fundamental issue that OFDM can't be utilized in higher transmission capacity applications. [6]The other few are high top to average force proportion which is answerable for impedance acquaintance and it is touchy with a transporter to recurrence balance and floats. OFDM is additionally increasingly inclined to be influenced by synchronization mistakes like transporter recurrence balance (CFO), timing counterbalance (TO). Numerous techniques are proposed to lessen CFO and TO in the OFDM framework, yet CFO and stage clamor are not assessed because of the nearness of warm commotion. Presently a-days we are proceeding onward to the fifth era of remote correspondence framework (5G), with a high information rate and better transmission capacity application. [7] The primary objective of $5 G$ is to carry adaptability to cell organize. Numerous administrations like Device-to-Device (D2D) or Machine-to-Machine is in progress. [8] Another innovation superior to OFDM is required to meet the fundamental needs of these innovations. The significant need in $5 \mathrm{G}$ is the ability to deal with high information rate wide data transfer capacity signals, give low dormancy transmission, quick exchanging among uplink and downlink and to be vitality effective. This prompted the necessity of another strategy with better recurrence and time control when contrast with that of OFDM.

[9]Summed up the Frequency Division Multiplexing (GFDM) technique that was found to meet the prerequisites of a group of people yet to come that is in 5G. It has numerous characters as that of OFDM. 


\section{Nonlinear Distortion Effects In Generalized Frequency Division Multiplexing}

This is more spurred than LTE versatile correspondence since clients are allotted, supposed as Physical Resource Blocks (PRB). GFDM is a non-symmetrical sign that is, there is no 90degree stage move. The couple of favorable circumstances of GFDM are high information rate, wide data transfer capacity signals, lower OOB radiation, higher power against CFO and testing time balance. Right now isn't rectangular. In GFDM one image is a mix of subcarriers and sub-images. [10]GFDM has a low ghostly thickness on account of the adaptability of the beat molding channel. Even though there are numerous points of interest in GFDM, still it has ISI and Inter-Carrier Interference (ICI). Right now timing mistake is not exactly the length of CP because of its non-symmetry. Because of the nearness of obstruction the channel estimation isn't minor.[11]GFDM is a square-based separated multi-bearer adjustment plot. This comprises of a lot of contiguous subcarriers and images. Here the roundabout channel is utilized to beat molded the subcarriers. By utilizing this strategy alongside windowing OOB can be decreased when contrasted with OFDM. The use of non-rectangular heartbeat forming is likewise an explanation behind the decrease of OOB, accordingly lessening the impedance in the framework. Because of the decrease of OOB, GFDM can be utilized for transporter conglomeration. The structure of GFDM signals is more modern than the current signs. The fundamental preferred position of GFDM is that it utilizes just a single cyclic prefix (CP) for a gathering of images as opposed to CP for one image as utilized in OFDM.The primary hindrance of the GFDM framework is that since it is a non-symmetrical framework because of the use of a model transmit channel the framework is progressively inclined towards Mean Square Error (MSE) and Symbol Error Rate (SER). The structure of the handset is a more unpredictable one than that of the OFDM framework.

\section{RELATED WORK}

\section{A. Minimum Mean Square Error}

Minimum Mean Square Error (MMSE) is an estimation method that is used to reduce mean square error (MSE). MSE is a common measure of estimator quality of the fitted values of a dependent variable.

$$
\mathrm{G}(\mathrm{k})=\mathrm{W}(\mathrm{kR}(\mathrm{k})
$$

Where,

\section{G(k)-Output of the MMSE filter}

$\mathrm{R}(\mathrm{k})$-Output of the communication system

\section{$\mathrm{W}(\mathrm{k})$-Weiner filter}

Then according to MMSE theory the error[S(k)-G(k)], it is orthogonal to $\mathrm{R}(\mathrm{k})$. [12]The term MMSE more specifically refers to estimation during a Bayesian setting with a quadratic cost function. The fundamental thought behind the Bayesian way to deal with estimation originates from reasonable circumstances where we as often as possible have some earlier data about the parameter to be evaluated.

\section{B. Extended Kalman Filter}

[13] Expanded Kalman Filter (EKF) is a nonlinear channel that linearizes about an estimation of the correct

mean and covariance. At this moment isn't basic that the state progress and discernment models are straight components of the state, rather it will in general be a differentiable limit.

$$
\begin{gathered}
\mathrm{Xk}_{\mathrm{k}}=\mathrm{f}\left(\mathrm{Xk}-1 \mathrm{Uk}_{\mathrm{k}}\right)+\mathrm{Wk}_{\mathrm{k}} \\
\mathrm{Zk}_{\mathrm{k}}=\mathrm{h}\left(\mathrm{Xk}_{\mathrm{k}}\right)+\mathrm{Vk}
\end{gathered}
$$

Where $\mathrm{Wk}$ and $\mathrm{Vk}$ are the processes

Perception clamors are thought to be with zero mean and covariance Qk and Rk. The burden of the Extended Kalman Filter is that it's anything but an ideal estimator it might likewise be an ideal estimator just when both the estimation and state progress model or direct. Another main problem is that the estimated covariance matrix tends to underestimate the true covariance matrix thereby increasing risk.

\section{Scatterd Pilot Channel}

Pilots are subcarriers which are used to track the residual phase error when it is present after frequency correction when the correction is not done the constellation point starts rotating either in a negative or positive angle. [14] The pilot sequence that is transmitted along with the data is the unmodulated data. These are used for synchronization and channel estimation purpose to improve the channel capacity the main thing to be concentrated is channel estimation. An expansion in the quantity of pilots will expand the effectiveness to appraise the channel.

\section{PROPOSED METHOD}

In the proposed method we combine the MF and the $\mathrm{ZF}$ techniques on the receiver side. As we discussed above the MF earlier, the advantage of the matched filter is made of use. $\mathrm{ZF}$ is used for interference cancellation and to reduce the long delays.

\section{A. Matched Filter}

Matched filter (MF) is a technique in which a correlation of the known signal with an unknown signal to recognize the unknown signals template. This infers the obscure sign is convolved with the conjugated time-switched form of the layout. The principle motivation behind the coordinated channel is to recognize the beat within the sight of added substance commotion. It is an ideal direct channel used to diminish clamor in this manner expanding the sign to commotion proportion. It is better for the sign which has added substance stochastic commotion.

Output of matched filter can be given by,

$$
\mathrm{Y}(\mathrm{t})=\mathrm{g}(\mathrm{t}) * \mathrm{~h}(\mathrm{t})+\mathrm{w}(\mathrm{t}) * \mathrm{~h}(\mathrm{t})
$$

Where,

$$
\text { g(t)-Input pulse signal }
$$

$\mathrm{h}(\mathrm{t})$-Matched filter function

$\mathrm{w}(\mathrm{t})$-White gaussian noise

This equation can also be written as

$$
\mathrm{Y}(\mathrm{t})=\mathrm{g} 0(\mathrm{t})+\mathrm{n}(\mathrm{t})
$$

$\mathrm{g} 0(\mathrm{t})$ represents signal power and $\mathrm{n}(\mathrm{t})$ represents noise.

The peak pulse SNR can be given by 
$\eta=$ Instantaneous power/Average power

$$
\eta=g^{2} / E\left\{n^{2}(t)\right\}
$$

where,

T-Symbol period.

\section{B. Zero Forcing}

Zero Forcing (ZF) is a versatile adjustment calculation wherein the equalizer coefficient is picked with the end goal that it powers the examples of the consolidated channel and equalizer motivation reaction to zero at wherever aside from one of the example focuses. This implies it applies the opposite of the channel recurrence reaction to the got signal. Along these lines, it reestablishes the first sign. It lessens Inter Symbol Interference (ISI) and furthermore self-impedance.

$$
\operatorname{Hch}(f) \operatorname{Heq}(f)=1,|f|<1 / 2 T
$$

Where,

$$
\begin{aligned}
& \text { Hch(f) - Folded frequency response of the channel } \\
& \text { Heq(f) - Frequency response of equalizer } \\
& \text { T- Symbol duration }
\end{aligned}
$$

Frequency response of the equalizer is periodic with a period equal to the symbol rate $1 / \mathrm{T}$.

The main disadvantage it works well for only noise-free systems. At certain frequencies the signal received maybe a week, to increase the magnitude of the signal, the gain of the filter is increased. This lifts up the commotion which prompts the obliteration of sign to clamor proportion.

\section{HPA}

The High Power Amplifier (HPA) is required on the transmitter to make the data sign can be transmitted to the most extreme with the goal that it can arrive at a more extensive zone. All in all, HPA will be worked in a straight locale moving toward the immersion district for expanding power effectiveness. When HPA input power rises near the immersion, it expands power proficiency and broadens the lifetime of the versatile transmitter battery. Be that as it may, HPA will create abundancy contortion if it's worked during this region. Nonlinear twisting gratitude to HPA causes a few impacts, both for yourself and different clients. Nonlinear bending impacts incorporate nonlinear mutilation, stage contortion and scattering dissipate plot. The adequacy contortion impact likewise creates consonant and entomb adjustment flags outside the waveband that prompts the transmitted spread of the ghastly sign so it disturbs the close by channels.

\section{GFDM Transmitter}

The proposed method consists of an ideal Transmitter with a channel coder followed by a subcarrier mapping. To avoid loss of data during transmission, a cyclic prefix is added at the end of each bit. Further, the data is passed into a digital pulse shaper to prevent the symbol from pulse broadening. This digital pulse shaped symbols are then converted from frequency domain to time domain followed by Digital to Analog conversion. A power amplifier is used to boost the strength of the signals.

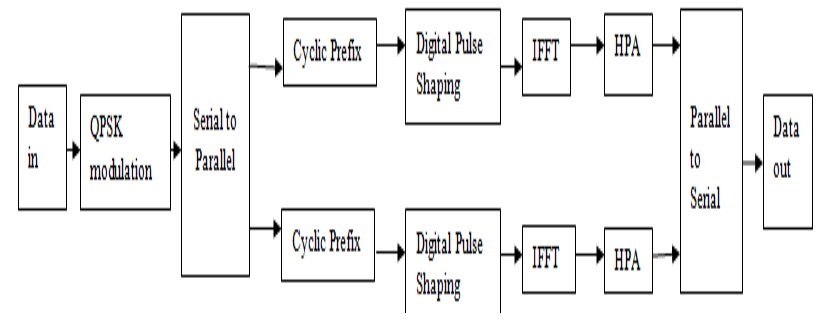

Fig.1.Block diagram of the transmitter

The input symbol is initially fed into the transmitter side in the form of bits which passes through a QPSK modulator. QPSK modulation is used to send a signal over a certain band pass frequency range resulting in providing each signal its frequency. Here, the function of QPSK is to transmit two bits per symbol to provide four possible separations of signals for easy transmission and helps to retrieve original information at the receiver side.

The next step of transmission involves the serial to parallel conversion. In this single input signal is split into multiple subcarrier signals. This is used to convert a stream of data elements received in a time sequence. In this paper, an input of 64 bits is divided into four 16 bits subcarriers. Each subcarrier is further carried out with the following processes.

The signal is further is added with a cyclic prefix to diminish the Inter Symbol Interference. The finish of every image is rehashed so the straight convolution displayed as roundabout convolution. This methodology obliges basic recurrence space preparing, for example, channel estimation and evening out and it is additionally used to improve heartiness to multipath engendering.

The yield from the cyclic prefix is gone through an advanced heartbeat shaper where the sign is liberated from the obstruction. While transmitting a signal at a high tweak rate through a band restricted channel can make a between image impedance. As the adjustment rate builds, the sign's data transmission increases than the channel transfer speed; the channel begins to acquaint bending with the sign. This contortion, for the most part, shows itself as between image impedance. This activity of presenting a computerized beat shaper is predominantly to lessen the between image obstruction. All the above procedure happens in the recurrence area. To transmit the signs to the recipient the signs must be changed over from recurrence area to time-space for which a reverse Fourier change happens. The procedure of backward Fourier change changes the signs from the recurrence area to time-space.

In IFFT the mind-boggling conjugate of the item is utilized. Rather than N modulators, one can perform IFFT on the signs to get the IDFT which is the examples of the adjusted transmit signals.

The last phase of transmission experiences a corresponding to sequential change where all the four sub transporter signals are consolidated to a solitary sign before arriving at the beneficiary. It changes over a surge of numerous information components that are gotten at the same time into a flood of information components transmitted in time arrangement. Thus, the signal with 64 bits undergoes all the above processes to prevent loss of data and reduce interference in the transmitter side. 


\section{Nonlinear Distortion Effects In Generalized Frequency Division Multiplexing}

This signal is then ready to process and transmit to the receiver. To determine the interference an additional noise is added along with the original input signal.

\section{E. GFDM Receiver}

The received signal comprises of the original input signal along with the added white Gaussian noise. The received signal is initially fed into a serial to parallel converter. As done earlier in the transmitter the reverse operation takes place. A single signal is divided and sent into multiple subcarrier signals. The function of this converter is to make the transmission of signals to individualusers easily.

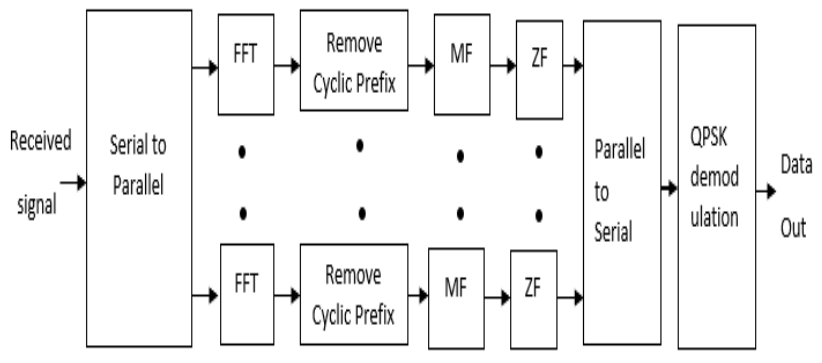

Fig.2. Block diagram of the receiver.

This is followed by a Fast Fourier Transform where the received signal is converted from Time-domain to Frequency domain. An FFT is a calculation that examples a sign over some undefined time frame and partitions it into its recurrence segments.

This part is single sinusoidal motions at particular frequencies each with their adequacy and stage.

Let $\mathrm{X} 0, \ldots \ldots, \mathrm{XN}-1$ be complex numbers. The DFT is defined by the formula

$$
\mathrm{Xk}=-\mathrm{i} 2 \prod \mathrm{kn} / \mathrm{N}
$$

The cyclic prefix added in the transmitter side is removed when reaching the receiver side. The main objective of this paper is to reduce interference on the receiver side. To reduce the interference a Matched Filter is added in the receiver. This Matched Filter is the ideal straight channel for augmenting the Signal to Noise proportion within the sight of clamor.

The channel input $\mathrm{x}(\mathrm{t})$ comprises of a heartbeat signal $\mathrm{g}(\mathrm{t})$ tainted by added substance channel clamor $\mathrm{w}(\mathrm{t})$, as appeared by

$$
x(t)=g(t)+w(t), 0 \leq t \leq T
$$

where,

$\mathrm{T}$ is a subjective perception interim.

The beat signal $\mathrm{g}(\mathrm{t})$ may speak to a twofold image I or 0 during an information correspondence framework. The $\mathrm{w}(\mathrm{t})$ is that the example capacity of a clamor procedure of zero mean and force unearthly thickness No/2.The source of uncertainty lies within the noise $\mathrm{w}(\mathrm{t})$. The function of the receiver is to detect the heartbeat signal $\mathrm{g}(\mathrm{t})$ in an optimum manner, given the received signal $x(t)$.

To satisfy this requirement, we've to optimize the planning of the filter so on minimize the consequences of noise at the filter output in some statistical sense and thereby enhance the detection of the heartbeat signal $g(t)$. Since the filter is linear, the resulting output $\mathrm{y}(\mathrm{t})$ may be expressed as

$$
y(t)=g 0(t)+n(t)
$$

where go(t) and $n(t)$ is produced by the signal and noise components of the input $\mathrm{x}(\mathrm{t})$, respectively.

A basic method for depicting the need that the yield segment go(t) be significantly more noteworthy than the yield commotion part $n(t)$ is to have the channel make the momentary force within the output go(t), measured at time $t$ $=\mathrm{T}$, as large as possible compared with the typical power of the output noise $n(t)$. This is like maximizing the height pulse signal-to-noise ratio, defined as

$$
\eta=\frac{|\mathrm{g} 0(\mathrm{~T})| 2}{\mathrm{E}[\mathrm{n} 2(\mathrm{t})]}
$$

This paper introduces an improved nonlinear parallel interference cancellation scheme for Generalized Frequency Division Multiplexing (GFDM) that significantly reduces the degrading effect on the specified user of interference from the other users that share the channel. The execution multifaceted nature of the plan is directly inside the number of clients and works on the very truth that multiprocessing all the while expels from every client an area of the obstruction created by the rest of the clients get to the channel the amount being corresponding to their dependability. The multiprocessing is often wiped out multiple stages. The proposed conspire utilizes provisional choice gadgets at the numerous phases to supply the first dependably assessed got information for age and crossing out of client obstruction. Recreation results are given for a wreck of different circumstances, particularly, those cases that the investigation is simply excessively perplexing. The inclusion of multistage parallel interference cancellation during a GFDM receiver can significantly improve its performance relative thereto of a standard GFDM receiver where no interference cancellation is attempted as compared with Serial Interference Cancellation, employing a PIC can reduce interference simultaneously which reduces time delay to an excellent extent.

The final stage involves parallel to a serial converter where all the subdivided signals are combined and sent as one single signal to the respective user.

\section{RESULTS AND DISCUSSION}

Simulation performed with GFDM following parameters: $\mathrm{K}=8$ sub-transporter, $\mathrm{M}=10$ sub-image, 16 QAM modulation mapping, utilizing the RRC with a move off calculate 0,3 as indicated in Table 1 . During this examination, we research the impact of abundancy mutilation on GFDM. 
Table I. Simulation Parameter

\begin{tabular}{|l|l|l|l|}
\hline Parameter & Notation & GFDM & OFDM \\
\hline $\begin{array}{l}\text { Sampling } \\
\text { frequency }\end{array}$ & Fs & 16 & 16 \\
\hline Sampling count & K & 16 & 16 \\
\hline Samples per block & N & 10 & 16 \\
\hline Samples per block & M & 32 & 1 \\
\hline Pulse shape & G & RRC & Rectangular \\
\hline Roll of factor & A & 1 & . \\
\hline Mapping & A & 16OQAM & 16QPSK \\
\hline
\end{tabular}

Figure 3 compares the performance of OFDM and GFDM with the nonlinear interference passed with the configuration presented in Table 1. CCDF PAPR esteem for OFDM after given Non-Linear contortion has diminished from 20 to $16 \mathrm{~dB}$ so it diminished the BER execution.

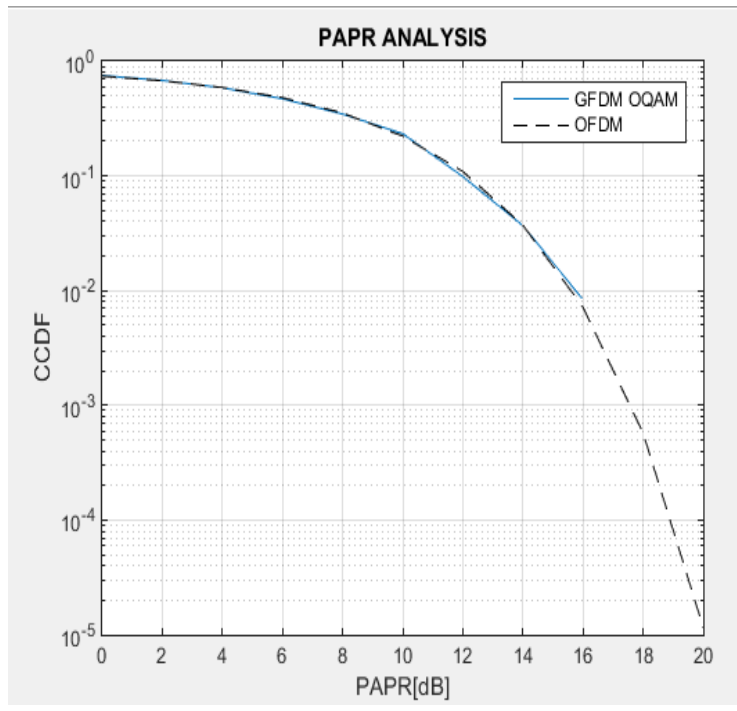

Fig3.PAPR Analysis

The spectrum of the signal after the amplitude distortion is that the distance between the side lobe becomes larger in comparison with the GFDM scheme generally. Therefore, the effect of the amplitude distortion can increase PSD. When viewed from the receiver, there's spectrum increasing in both scenarios due to out of band distortion. Figure 4 shows the facility spectrum of GFDM and OFDM.

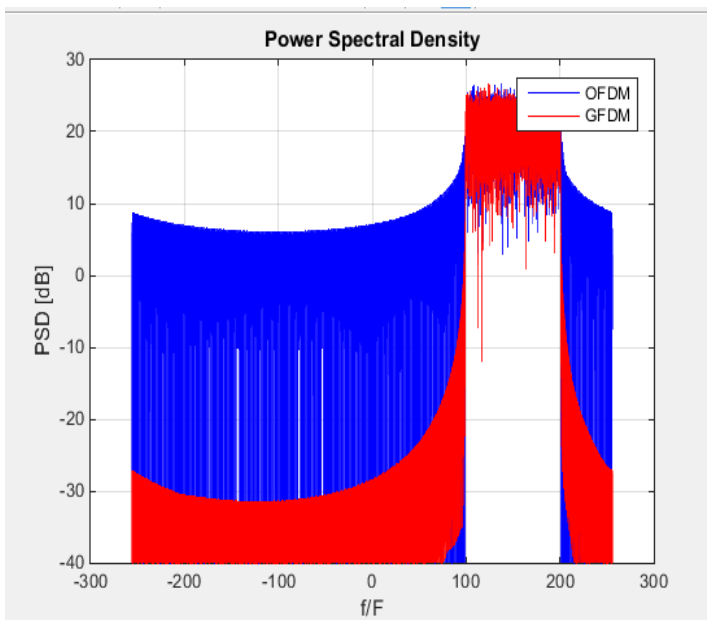

Fig4.Power spectrum
Figure 4 is used to find the strength of the signal at each frequency. This is used to find the frequency at which the signal can be sent with less noise and reduced interference within the information signal.

We analyze the performance of the GFDM with and without nonlinear distortion system in terms of BER versus SNR.

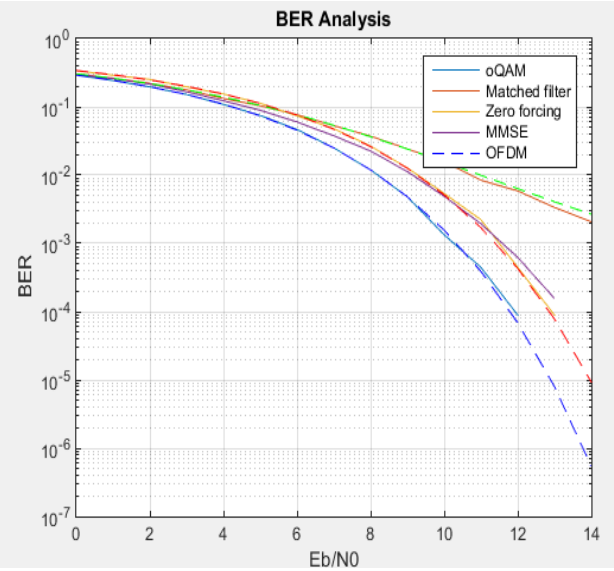

Fig5.BER Analysis

Figure 5 compares the performance of GFDM with and without the nonlinear interference passed on the AWGN or Rayleigh channel. When viewed from the influence of SNR (Signal to Noise Ratio) within the modeling of nonlinear distortions, if SNR is bigger so BER value tends to be smaller. Therefore the greater the noise, the SNR becomes smaller so that the bit error received within the receiver getting bigger. Firstly, from the observations of the three parameters previously, the amplitude distortion effects are increasing PAPR which can degrade the performance of GFDM. Secondly, raise the PSD of the signal, and therefore the third is the degradation of SER.

\section{CONCLUSION}

This paper reduces interference since it combines Matched Filter, Zero Forcing and HPA. By using Matched Filter, the noise that's added to the knowledge signal is reduced thereby increasing the Signal to Noise Ratio value. This study investigated and analyzed the effect of HPA amplitude distortion GFDM MIMO on the form of the spectrum, the PAPR, and therefore the BER within the AWGN channel. The system with HPA was appeared to have better range parameters, great PAPR, and lower BER values. Plenty fulness bending brought about by the HPA memory which worked inside the immersion district. the transmitter handling strategy decreases the effects of sufficiency contortion.

\section{ACKNOWLEDGMENT}

We would like to thank our guide Dr.S.Sumathi, who guided us in the right way, providing us the confidence that we can do our best in the project and the belief she had on us. We would also like to thank our other staff members who helped in the project work.

\section{Published By:}

Blue Eyes Intelligence Engineering

\& Sciences Publication

(C) Copyright: All rights reserved. 


\section{Nonlinear Distortion Effects In Generalized Frequency Division Multiplexing}

\section{REFERENCES}

1. Ari Endang Jayati, Wirawan, Titiek Suryani, Endroyono, "Characteristic of HPA Nonlinear Distortion Effects in MIMO-GFDM Systems," 978-1-5386-5041-7/18/\$31.00 @2018 IEEE.

2. Maximilian Matthe, Dan Zang and Gerhard Fettweis, "Low Complexity Iterative MMSE-PIC Detection for MIMO- GFDM," IEEE Transaction on Communication, pp. 1-30, 2017.

3. Shravan Kumar Bandari, V. V. Mani and A. Drosopoilos, "Convolutional codes for MGFDM system," IEEE Conference, pp 545-550, 2017.

4. Ahmad Nimr, Maximilian Matthé, Dan Zhang, and Gerhard Fettwei, "Optimal Radix-2 FFT Compatible Filters for GFDM," IEEE Communication Letters, vol. 21, no. 7, pp. 1497-1500, July 2017.

5. Seungyul Han, Youngchul Sung, and Yong H. Lee, "Filter Design for Generalized Frequency-Division Multiplexing, " IEEE Transactions on Signal Processing, vol. 65, no. 7, pp. 1644-1659, April 1, 2017.

6. Liang Chang, Geoffrey Ye Li, Jingchun Li, and Rong Li, "Blind Parameter Estimation of GFDM Signals Over Frequency-Selective Fading Channels," IEEE Transactions on Communication, vol. 64, no.3, pp. 1120-1131, March 2017.

7. Arman Farhang, Nicola Marchetti, and LindaE. Doyle, "Low-Complexity Modem Design for GFDM," IEEE Transactions on Signal Processing, vol. 64, no. 6, pp. 1507-1518, March 15, 2016.

8. Peng Wei, Xiang-Gen Xia, Yue Xiao, and Shaoqian Li, "Fast DGT-Based Receivers for GFDM in Broadband Channels," IEEE Transactions on Communication, vol. 64, no. 10, pp. 4331-4345, October 2016.

9. David W. Lin and Po-Sen Wang, "On the Configuration-Dependent Singularity of GFDM Pulse-Shaping Filter Banks," IEEE Communication Letter, vol. 20, no. 10, pp. 1975-1978, October 2016.

10. Zhipeng Zhong and Junqi Guo, " Bit Error Rate Analysis of MIMO-Generalized Frequency Division Multiplexing Scheme for 5th Generation cellular systems," IEEE International Conference on Electronic Information and Communication Technology, pp. 62-68, 2016.

\section{AUTHORS PROFILE}

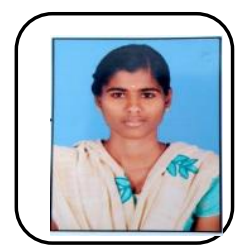

M.Kaviya, received her B.E degree in Electronics and Communication Engineering from Sri Ramakrishna Institute of Technology, India in 2018. She is pursuing M.E Communication Systems in Electronics and Communication Engineering from Adhiyamaan College of Engineering, India. Her research includes wireless communication.

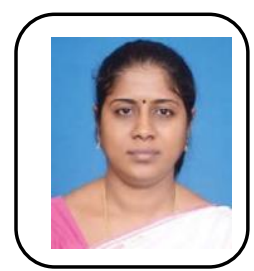

Dr. S.Sumathi, received $\mathrm{PhD}$ degree in information and Communication Engineering from Anna university, Chennai, India in 2009; ME degree in Applied Electronics from Anna University, Chennai, India in 2004 and BE degree in Electronics and Communication Engineering from Bharathiyar University, Tamilnadu, India in 1994. She is now working as Professor and Head, Department of Electronics and communication Engineering, Adhiyamaan College of Engineering, hosur, Tamilnadu. She has published more than 25 papers in various International Journals and Conference proceedings. 